\title{
A Rare Initial Presentation of Leukemia With Pneumocystis Jirovecii Pneumonia
}

\author{
Harmeen Goraya ${ }^{a}$ e, Makardhwaj Shrivastava ${ }^{\mathrm{b}}$, Olufisayo Otusanya ${ }^{\mathrm{b}}$, Amara Nandikolla ${ }^{\mathrm{c}}$, \\ Amit S. Tibb ${ }^{\mathrm{d}}$
}

\begin{abstract}
Pneumocystis jirovecii pneumonia (PJP) causes life-threatening respiratory failure in immunocompromised patients such as patients with HIV infection, hematologic and solid malignancies, organ transplantations, and autoimmune diseases and patients receiving immunosupressive medications. In HIV patients, PJP usually takes an indolent course while in HIV negative but immunocompromised patients, the disease is usually severe and is associated with a high mortality rate. A high index of suspicion and early institution of therapy is warranted. We present a case of life-threatening PJP as the first manifestation in a patient with undiagnosed acute leukemia.
\end{abstract}

Keywords: Opportunistic infection; Immunosuppression; Acute myeloid leukemia; Pneumocystis jirovecii pneumonia

\section{Introduction}

Pneumocystis jirovecii pneumonia (PJP) is an opportunistic infection caused by Pneumocystis jirovecii, an atypical fungus with a predilection for human lungs [1]. P. jirovecii causes infection in immunocompromised patients such as patients with AIDS, malignancies, organ transplantations, and autoimmune diseases and recipients of immunosuppressive medications [2]. The severity of the clinical manifestations of PJP varies, notably, according to HIV status. PJP may present as a life-threat-

Manuscript accepted for publication March 24, 2016

aDivision of Pulmonary and Critical Care, Albert Einstein College of Medicine/Montefiore Medical Centre, Bronx, NY 10461, USA

${ }^{b}$ Department of Internal Medicine, Albert Einstein College of Medicine/Jacobi Medical Center, Bronx, NY 10461, USA

'Division of Hematology and Oncology, Albert Einstein College of Medicine/ Montefiore Medical Center, Bronx, NY 10461, USA

dDivision of Pulmonary and Critical Care, Albert Einstein College of Medicine/Jacobi Medical Center, Bronx, NY 10461, USA

${ }^{\mathrm{e} C o r r e s p o n d i n g ~ A u t h o r: ~ H a r m e e n ~ G o r a y a, ~ D i v i s i o n ~ o f ~ P u l m o n a r y ~ a n d ~ C r i t i c a l ~}$ Care, Albert Einstein College of Medicine/Montefiore Medical Centre, Bronx, NY 10461, USA. Email: harmeen_5@yahoo.com

doi: http://dx.doi.org/10.14740/jmc2472w ening disease in immunocompromised HIV negative patients. We report a case of PJP presenting as a life-threatening illness in a previously undiagnosed and untreated leukemic patient.

\section{Case Report}

A 40-year-old Hispanic gentleman with well-controlled type II diabetes mellitus and hypertension presented with dyspnea, pleuritic chest pain, and cough which was occasionally productive of blood streaked, mucoid, and non-foul smelling expectoration of 3 days duration. He had been in his usual state of health and was actively working as a clerk prior to onset of symptoms. He denied fever, chills, malaise, palpitations, skin rash, bone pain or night sweats. He did not have preceding flulike symptoms. He denied history of tuberculosis or chronic respiratory conditions in the past, any sick contacts, allergies or recent travels and had no addictions. He lived with his family, denied unsafe sexual activity and had been living in USA since childhood. Family history was not contributory.

Vital signs on presentation were temperature of $98.3^{\circ} \mathrm{F}$, pulse rate of 106 beats per minute, respiratory rate of 36 and a blood pressure of 150/101 $\mathrm{mm} \mathrm{Hg}$. His oxygen saturation was $93 \%$ on room air. He had mild central pallor and multiple enlarged 1 - $2 \mathrm{~cm}$ sized, mobile, non-tender, rubbery lymph nodes

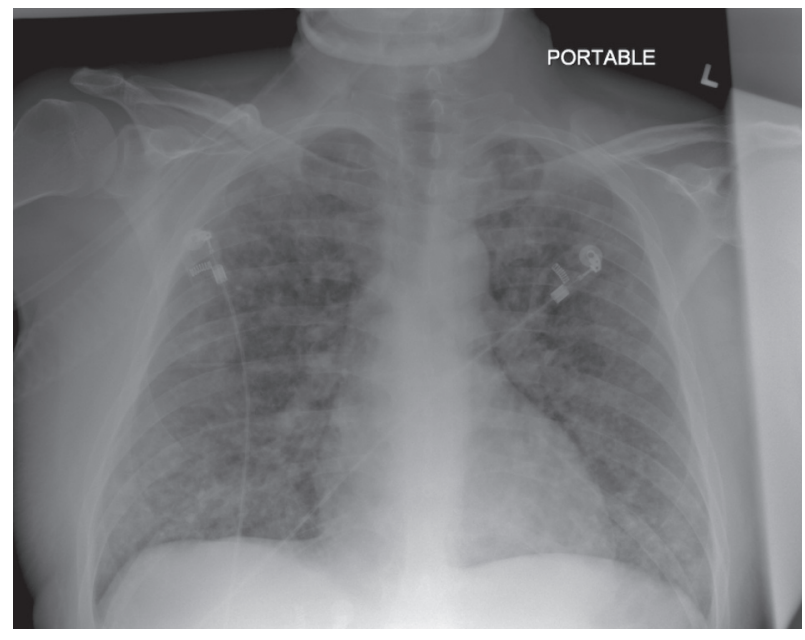

Figure 1. Chest $\mathrm{X}$-ray film showing diffuse bilateral interstitial infiltrates. 


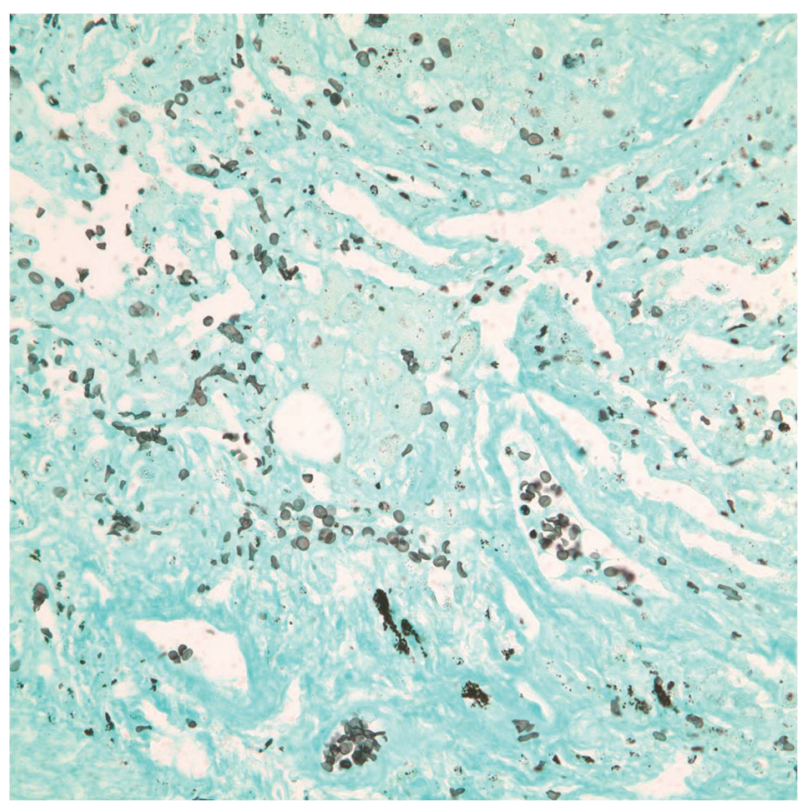

Figure 2. P. jirovecii on Gomori methenamine silver (GMS) stain.

were palpable in neck, supraclavicular region, axilla and inguinal areas. Mucosal petechial rash was noticed on oral exam in the oropharynx. There was mild sternal tenderness. There no cyanosis, clubbing or pedal edema, no skin rash or organomegaly. His work of breathing continued to increase requiring intubation for respiratory failure and intensive care unit (ICU) admission. Initial arterial blood gas showed $\mathrm{pH}$ of 7.453, $\mathrm{PCO}_{2}$ of $31.1 \mathrm{~mm} \mathrm{Hg}$, and $\mathrm{PO}_{2}$ of $70.3 \mathrm{~mm} \mathrm{Hg}$ on $100 \%$ non-rebreathing mask. Hemoglobin was $10.6 \mathrm{~g} / \mathrm{dL}$ with leukocyte count of $21.8 \times 10^{9} / \mathrm{L}$ and platelets of $27 \times 10^{9} / \mathrm{L}$. Peripheral smear revealed $11 \%$ segments, $2 \%$ bands, $14 \%$ lymphocytes, $40 \%$ monocytes, eosinophil $1 \%, 10 \%$ atypical lymphocytes, $5 \%$ pro-monocytes and $18 \%$ blasts. Liver and renal function tests were normal. Blood cultures drawn prior to antibiotics were negative for any bacterial or fungal growth. HIV test was negative. Chest radiograph revealed diffuse reticulonodular interstitial infiltrates in both lung fields (Fig. 1). Sputum smears for acid-fast bacilli (AFB) as well as induced sputum for $P$. jirovecii were negative. Bronchoscopy was subsequently performed. Broncho-alveolar lavage (BAL) and trans-bronchial lung biopsies were done. BAL gram stain, AFB and smears for $P$. jirovecii were negative. Cytology was negative for malignant or leukemic cells. Bacterial, mycobacterial, viral and fungal cultures were negative. Lung biopsy was negative for leukemic infiltrates but showed abundant $P$. jirovecii on Gomori methenamine silver stain (Fig. 2).

Given the peripheral smear findings and high clinical suspicion with no other risk factors to explain $P$. jirovecii infection, a bone marrow biopsy (BMB) was performed which revealed acute myeloid leukemia (AML, subtype M4eo) with high cellularity and complex cytogenetics (inv(16)) on immunophenotyping (Fig. 3).

On admission, patient was initiated on vancomycin and piperacillin/tazobactam for broad-spectrum coverage in addition to trimethoprim/sulfamethoxazole and steroids given high

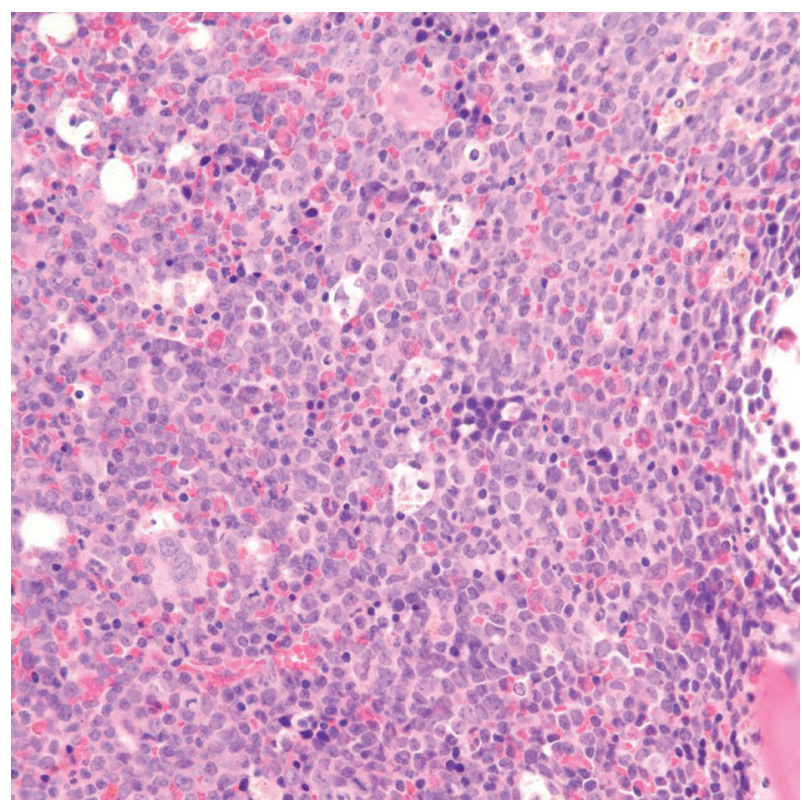

Figure 3. Bone marrow biopsy slide showing AML with marked hypercellularity.

index of suspicion for PJP. After clinical stabilization and confirmation of diagnosis of AML, induction chemotherapy with arabinoside-C and idarubicin was initiated. Neupogen was administered for severe neutropenia with absolute neutrophil count of $0.03 \times 10^{9} / \mathrm{L}$. He subsequently developed acute respiratory distress syndrome (ARDS) and tumor lysis syndrome resulting in prolonged ventilator support. Patient also developed acute kidney injury requiring renal replacement therapy which resulted in gradual improvement of renal function. Patient continued to improve and was eventually weaned off the ventilator and extubated 29 days later. He was subsequently discharged to an acute rehabilitation facility with outpatient follow-up in the hematology clinic.

\section{Discussion}

PJP is a significant cause of morbidity and mortality in immunocompromised patients. PJP has been well studied in HIV patients and is categorized as an AIDS defining illness. Due to the availability of effective highly active antiretroviral therapy and use of PJP prophylaxis in HIV patients with CD4 count less than $200 / \mu \mathrm{L}$, the incidence of PJP in HIV patients has been declining [3]. However, in HIV negative immunocompromised patients, the incidence of $P$. jirovecii infection is reported to be increasing [4]. Compared to HIV positive patients, $P$. jirovecii infection in HIV negative, immunosuppressed patients tend to take a fulminant form with rapid progression into life-threatening respiratory failure requiring ventilator support and ICU care $[1,5]$. Delay in commencement of therapy for PJP in this subset of population is associated with poor outcomes. Mortality rate as high as $59 \%$ has been reported [6]. Physicians need to have a high index of clinical suspicion and early institution of therapy is hence warranted in these patients and can be life 
saving, as was seen in our patient.

To the best of our knowledge, de novo leukemia (untreated) presenting with acute life-threatening PJP has not been reported previously. Our case is also interesting since AML with chromosome 16 inversions is a rare type of high risk leukemia [7] (AML with inv(16)). Multiple reports have suggested an increased risk of pulmonary complications associated with AML and inv(16) compared to AML and no inv(16) [8]. In most of these cases, an etiology was not identified. Whether AML with inv(16) predisposes to increased severity and susceptibility to opportunistic infections needs to be studied further.

\section{Conclusion}

Undiagnosed/untreated acute leukemia presenting initially as severe $P$. jirovecii is exceedingly rare. Patients with AML with inv(16) are prone to pulmonary complications. PJP in this subset of population is life-threatening. A high index of suspicion and early initiation of therapy in a monitored setting can be life saving.

\section{References}

1. Thomas CF, Jr., Limper AH. Pneumocystis pneumonia. $\mathrm{N}$ Engl J Med. 2004;350(24):2487-2498.

2. Bollee G, Sarfati C, Thiery G, Bergeron A, de Miranda
S, Menotti J, de Castro N, et al. Clinical picture of Pneumocystis jiroveci pneumonia in cancer patients. Chest. 2007;132(4):1305-1310.

3. Huang L, Cattamanchi A, Davis JL, den Boon S, Kovacs J, Meshnick S, Miller RF, et al. HIV-associated Pneumocystis pneumonia. Proc Am Thorac Soc. 2011;8(3):294300 .

4. Catherinot E, Lanternier F, Bougnoux ME, Lecuit M, Couderc LJ, Lortholary O. Pneumocystis jirovecii Pneumonia. Infect Dis Clin North Am. 2010;24(1):107-138.

5. Monnet X, Vidal-Petiot E, Osman D, Hamzaoui O, Durrbach A, Goujard C, Miceli C, et al. Critical care management and outcome of severe Pneumocystis pneumonia in patients with and without HIV infection. Crit Care. 2008;12(1):R28.

6. Li MC, Lee NY, Lee CC, Lee HC, Chang CM, Ko WC. Pneumocystis jiroveci pneumonia in immunocompromised patients: delayed diagnosis and poor outcomes in non-HIV-infected individuals. J Microbiol Immunol Infect. 2014;47(1):42-47.

7. Sartor C, Papayannidis C, Chiara Abbenante M, Curti A, Polverelli N, Ottaviani E, Iacobucci I, et al. A case report of acute myeloid leukemia and neurofibromatosis 1 . Hematol Rep. 2013;5(2):28-29.

8. Perez-Zincer F, Juturi JV, Hsi ED, Hoeltge GA, Rybicki LA, Kalaycio ME. A pulmonary syndrome in patients with acute myelomonocytic leukemia and inversion of chromosome 16. Leuk Lymphoma. 2003;44(1):103-109. 